\title{
CONFÉRENCE
}

\section{Les complications des pathologies infectieuses de la face Dr. LUTZ Jean-Christophe}

Chirurgien Maxillo-Facial

Maître de conférence des Universités - Praticien Hospitalier - CHU Strasbourg

Les complications infectieuses de la face sont extrêmement fréquentes dans la pratique quotidienne. Si la porte d'entrée peut être cutanée, l'étiologie dentaire est toutefois prédominante. La prescription intempestive d'AINS ou l'automédication sont des facteurs favorisants établis mais encore couramment rencontrés. Malgré la banalité de ces infections, leur pronostic n'en est pas moins potentiellement tragique, engageant parfois le pronostic vital. La séméiologie et les critères de gravité de ces diverses complications infectieuses doivent donc être parfaitement connus de tout chirurgien oral. À cette fin, nous rappelons le tableau clinique de différents types d'infections, notamment des cellulites faciales et de leurs complications évolutives, la thrombophlébite crânio-faciale, la médiastinite mais aussi celui des sinusites maxillaires et des complications systémiques. Nous illustrons les principes de leur traitement.

Une collaboration optimisée entre le chirurgien-dentiste, le chirurgien oral et le chirurgien maxillofacial serait en mesure de restreindre la prévalence de ces complications graves. 